\title{
Simulation Study for the Sensitivity and Mean Sojourn Time Specific Lead Time in Cancer Screening When Human Lifetime is a Competing Risk
}

Sarah K. Kendrick, Shesh N. Rai and Dongfeng Wu*

Department of Bioinformatics and Biostatistics, University of Louisville, Louisville, $K Y$, USA

\begin{abstract}
Purpose: The purpose of this paper is to examine the sensitivity and mean sojourn time specific lead time distribution in cancer screening trials when lifetime is a random variable in order to explore possible optimal initial age at screening and screening frequency.

Methods: Summarized methods from Wu et al. (2012). Simulation was used in order to estimate the distribution of the lead time for a hypothetical individual with a future screening schedule. The lifetime distribution used comes from the Social Security Administration's actuarial life tables. The lead time distribution was then calculated based on a loglogistic sojourn time distribution with two mean sojourn times (2, and 10 years), using three different initial screening ages, $\mathrm{t}_{0}=40,50,60$, different screening sensitivities ( 0.3 and 0.5 for men; 0.8 and 0.9 for women), and two different screening frequencies, one and two years for both men and women.
\end{abstract}

Results: Smaller time intervals between screenings yield a smaller probability of no early detection and a greater expected lead time.

Keywords: Cancer; Early detection; Periodic screening; Sojourn time; Sensitivity; Lead time

\section{Introduction}

Cancer has been sweeping the nation in recent history, and there has been a lot of research on cancer treatments and effective screening for cancer. Among men, prostate cancer is the most common cancer followed by lung cancer and colorectal cancer with lung cancer being the leading cause of cancer death in men followed by prostate cancer. For women, the most prevalent cancers are breast cancer, followed by lung cancer, and then colorectal cancer with lung cancer being the leading cause of cancer death, followed by breast cancer [1]. Cancer screening methods are implemented in the hopes of detecting cancer early which will give an individual a longer lead time and, hopefully, a better prognosis.

The most common model for the progression of cancer is one occurring in three stages: $S_{0}, S_{p}$, and $S_{c}[2] . S_{0}$ is known as the disease-free state, $S_{p}$ is the preclinical state in which the individual has developed the disease, and can be detected by screening, but has shown no clinical symptoms, and $\mathrm{S}_{c}$ is the clinical state in which the individual exhibits clinical symptoms. The difference in time between the age at which an individual enters the preclinical state, $t_{p}$ and the age the individual begins to experience clinical symptoms, $t_{c},(>t)$ is defined as the individual's sojourn time. If disease was detected by screening after the individual entered the preclinical state, but prior to experiencing any symptoms, the time difference between the age at detection by screening and the age at onset of symptoms is referred to as the individual's lead time (Figure 1a).

The topic of lead time has been an area of much research over the past 20 to 30 years. Many studies have worked to determine the parameters, formulas, and distributions that most accurately estimate the important descriptive statistics for the lead time distribution, such as mean and variance. One of the major contributions to the topic of lead time was the work done by Prorok in 1982. Prorok's goal was to determine the optimal number of screenings required for effective and efficient evaluation in repetitive screening trials. He used the lead time properties of the $\mathrm{i}^{\text {th }}$ screen detected cases to develop a stopping rule for these kinds of studies. What he found was that the lead time tended to stabilize after four to five screenings when the screening frequency was held constant. This property showed that any screenings in excess of the fourth or fifth one may no longer provide any additional information [3]. However, Prorok's design contained one major limitation: he derived the distribution of the lead time for cases detected by the $\mathrm{i}^{\text {th }}$ exam only, without checking into the distribution of the lead time for the screen-detected cohort as a whole population, and he did not take interval cases, where lead time is zero, into account [3]. Wu et al. used a different approach by calculating probability directly for cases when the lead time is positive or zero, they derived the lead time distribution for the whole cohort including both screen-detected cases and interval cases. The procedure is greatly simplified without using the forward and backward recurrence times, and their work included Prorok's results as a special case. However, $\mathrm{Wu}$ et al. derived the lead time distribution assuming that human lifetime is a fixed value, which is unrealistic. Then, $\mathrm{Wu}$ et al. extended their previous method to the case where lifetime is subject to competing risks, and is, therefore, a random variable.

In this paper we will carry out extensive simulations based on the methods developed by $\mathrm{Wu}$ et al. in 2012. Wu et al simulated data only for women and breast cancer; we extend their work to examine a

${ }^{*}$ Corresponding author: Dongfeng $\mathrm{Wu}$, Department of Bioinformatics and Biostatistics, University of Louisville, Louisville, KY, USA, Tel: 502-852-1888 E-mail: Dongfeng.Wu@Louisville.Edu

Received August 22, 2015; Accepted September 05, 2015; Published September 14,2015

Citation: Kendrick SK, Rai SN, Wu D (2015) Simulation Study for the Sensitivity and Mean Sojourn Time Specific Lead Time in Cancer Screening When Human Lifetime is a Competing Risk. J Biom Biostat 6: 247. doi:10.4172/21556180.1000247

Copyright: (C) 2015 Kendrick SK, et al. This is an open-access article distributed under the terms of the Creative Commons Attribution License, which permits unrestricted use, distribution, and reproduction in any medium, provided the original author and source are credited. 
broader spectrum of simulations that can be applied to several different cancers (see motivating example). We look at three hypothetical cohorts of initially asymptomatic men and women. The three cohorts will be based on initial age at screening: 40,50 , and 60 years old. Within each cohort we will examine the effect of two different sensitivities, 0.3 and 0.5 for men, 0.8 and 0.9 for women as well as two different screening frequencies and two different mean sojourn times on the lead time distribution: screening interval $\delta=1,2$ years and mean sojourn times 2 and 10 years. We will use the log-logistic distribution as the sojourn distribution. Our results will give insight into the benefits of screening for different types of cancers, as well as the ideal initial screening age and screening frequency.

\section{Motivating Example}

Breast, lung, prostate, and colorectal cancers are the four most prevalent cancers for men and/or women in the United States. Each of these four cancers has a different screening method(s) used for early detection which could lead to a better prognosis. Prostate cancer has two different screening methods, a digital rectal exam (DRE) and a prostate specific antigen test (PSA). However, the United States Preventative Services Task Force does not recommend getting the PSA test for men who are asymptomatic [4]. Screening options for breast cancer consist of self-exams, mammograms, and clinical breast exams. Lumps and changes in size or shape of the breast(s) or underarm are warning signs for breast cancer, and are usually detected by self

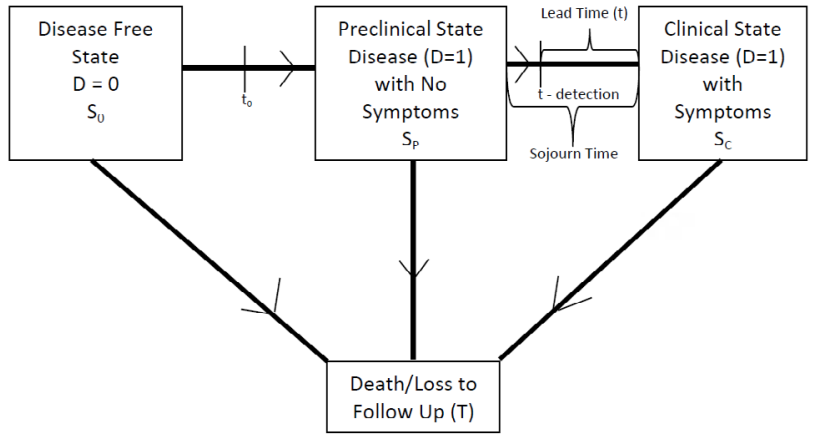

Figure 1a: Disease progression model and screening process.

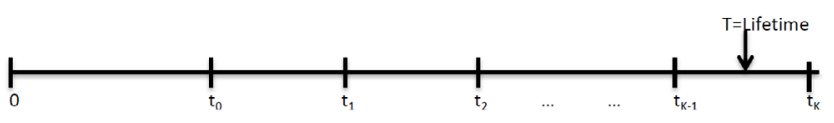

Figure 1b: Screening timeline. or clinical examination by a nurse or doctor. Even though these two methods can be helpful in detecting breast cancer, they have not been shown to decrease one's risk of death from breast cancer [5]. However, getting regular mammograms, an X-ray of the breasts, has been shown to decrease an individual's risk of death due to breast cancer [5]. Screening options for lung cancer include chest X-rays, sputum cytology, and CT scans but there is debate over whether any of these actually help in decreasing deaths from lung cancer [6]. Colorectal cancer, liver cancer, and all other cancers have screening test(s) just as the cancers mentioned above.

Each screening method has a different sensitivity and each cancer a different mean sojourn time and median age at diagnosis (Table 1). The sensitivity of a screening method is defined as the proportion of diseased individuals that test positive for disease during screening. A higher screening sensitivity is better - the screening method has a higher probability of detecting disease when disease is actually present. Sensitivity plays a crucial role in screening. At younger ages, when disease prevalence is low, sensitivity is also lower and screening does not need to occur as frequently. However, as individuals get older, disease prevalence and probability increase as does screening sensitivity and screening should be done more frequently. Therefore it is important to examine the effect of different screening sensitivities on the lead time distribution in cancer screening. We summarized $\mathrm{Wu}$ et al. methods and extended their work to study the effect of sensitivity and mean sojourn time on the lead time distribution. Wu et al. applied their methods only to breast cancer screening. Extending this to include a variety of screening sensitivities and mean sojourn times allows us to apply our results to several different cancers that have not been studied before.

\section{Methods}

\section{Summary}

$\mathrm{Wu}$ et al. developed methods for estimating the lead time distribution when life time is a random variable. These methods were an extension of a previous paper published in 2007 by Wu et al., where they estimated the lead time distribution when life time was fixed. Their methods showed that the lead time distribution is a mixture consisting of two parts: a point mass at zero, $\mathrm{P}(\mathrm{L}=0 \mid \mathrm{D}=1, \mathrm{~T}=\mathrm{t})$, indicating an interval case, and a piece-wise continuous conditional probability density function, $f_{L}(z \mid D=1, T=t)$, where $L$ is the lead time, $D$ is true disease status, and $\mathrm{T}$ is lifetime, a random variable:

$$
\begin{aligned}
& P\left(L=0 \mid D=1, T \geq t_{0}\right)=\int_{t_{0}}^{\infty} P(L=0 \mid D=1, T=t) f_{T}\left(t \mid T \geq t_{0}\right) d t \\
& f_{L}\left(z \mid D=1, T \geq t_{0}\right)=\int_{t_{0}+z}^{\infty} f_{L}(z \mid D=1, T=t) f_{T}\left(t \mid T \geq t_{0}\right) d t \quad z \in(0, \infty)
\end{aligned}
$$

\begin{tabular}{|c|c|c|c|c|c|c|}
\hline Cancer Type & Screening Method & Gender & $\begin{array}{c}\text { Median age at } \\
\text { diagnosis (years) }\end{array}$ & $\begin{array}{c}\text { Sensitivity } \\
\text { (Confidence Interval) }\end{array}$ & $\begin{array}{l}\text { Mean Sojourn Time } \\
\text { (range, years) }\end{array}$ & References \\
\hline Lung & Chest X-Ray & $\mathrm{M} / \mathrm{F}$ & 70 & $0.89(0.72,0.98)$ & $1.38-3.86$ & {$[7,8]$} \\
\hline Colorectal & $\begin{array}{l}\text { Colonoscopy } \\
\text { FOBT }^{\mathrm{a}}\end{array}$ & $\mathrm{M} / \mathrm{F}$ & 68 & $\begin{array}{l}0.75(0.45,0.97) \\
0.75(0.65,0.84)\end{array}$ & $2.2-3.9$ & [7,9-11] \\
\hline Breast & Mammogram & $\mathrm{F}$ & 61 & $0.74(0.43,0.97)$ & $1.9-3.1$ & {$[7,12,13]$} \\
\hline Prostate & $\begin{array}{l}\mathrm{PSA}^{\mathrm{b}} \\
\mathrm{DRE}^{\mathrm{c}}\end{array}$ & M & 66 & $\begin{array}{c}0.21(-,-) \\
0.56(0.53,0.59)\end{array}$ & $11.3-12.6$ & {$[7,14,15]$} \\
\hline
\end{tabular}

aFecal occult blood test

${ }^{b}$ Prostate specific antigen

'Digital rectal exam

Table 1: Screening sensitivities, mean sojourn time, and age at diagnosis for four cancers. 
where,

$$
\begin{aligned}
& \mathrm{f}_{\mathrm{T}}\left(\mathrm{t} \mid \mathrm{T} \geq \mathrm{t}_{0}\right)= \begin{cases}\frac{\mathrm{f}_{\mathrm{T}}(\mathrm{t})}{\mathrm{P}\left(\mathrm{T}>\mathrm{t}_{0}\right)}=\frac{\mathrm{f}_{\mathrm{T}}(\mathrm{t})}{1-\mathrm{F}_{\mathrm{T}}\left(\mathrm{t}_{0}\right)}, & \text { if } t \geq \mathrm{t}_{0} \\
0, & \text { otherwise. }\end{cases} \\
& \mathrm{P}(\mathrm{L}=0 \mid \mathrm{D}=1, \mathrm{~T}=\mathrm{t})=\frac{\mathrm{P}(\mathrm{L}=0, \mathrm{D}=1 \mid \mathrm{T}=\mathrm{t})}{\mathrm{P}(\mathrm{D}=1 \mid \mathrm{T}=\mathrm{t})}, \\
& \mathrm{f}_{\mathrm{L}}(\mathrm{z} \mid \mathrm{D}=1, \mathrm{~T}=\mathrm{t})=\frac{\mathrm{f}_{\mathrm{L}}(\mathrm{z}, \mathrm{D}=1 \mid \mathrm{T}=\mathrm{t})}{\mathrm{P}(\mathrm{D}=1 \mid \mathrm{T}=\mathrm{t})},
\end{aligned}
$$

For a person currently at age $t_{0}$, the number of screening exams, $K$, is changing with his/her lifetime, so $\mathrm{K}$ is a random variable. However, if $s /$ he plans to follow a future screening schedule: $t_{0}<t_{1}<\ldots$, then $K=n$ if $\mathrm{t}_{\mathrm{n}-1}<\mathrm{T}<\mathrm{t}_{\mathrm{n}}$. When one's lifetime $\mathrm{T}$ falls in the interval $\left(\mathrm{t}_{\mathrm{K}-1}, \mathrm{t}_{\mathrm{K}}\right)$, we let $\mathrm{T}=\mathrm{t}_{\mathrm{K}}$ for simplification in formula expression (Figure 1b); in that case, the numerator and denominator in equations (4) and (5) can be expressed as:

$$
\begin{aligned}
P(L=0, D & \left.=1 \mid T=t_{K}\right) \\
& =\sum_{j=1 i=0}^{K} \sum^{j-1}\left(1-\beta_{i}\right) \ldots\left(1-\beta_{j-1}\right) \int_{t_{i-1}}^{t_{i}} w(x)\left[Q\left(t_{j-1}-x\right)-Q\left(t_{j}-x\right)\right] d x \\
& +\int_{t_{j-1}}^{t_{j}} w(x)\left[1-Q\left(t_{j}-x\right)\right] d x,
\end{aligned}
$$

and,

$$
\begin{aligned}
\mathrm{f}_{\mathrm{L}}\left(\mathrm{z}, \mathrm{D}=1 \mid \mathrm{T}=\mathrm{t}_{\mathrm{K}}\right) & \\
& =\sum_{\mathrm{i}=1}^{\mathrm{j}-1} \beta_{\mathrm{i}}\left\{\sum_{\mathrm{r}=0}^{\mathrm{i}-1}\left(1-\beta_{\mathrm{r}}\right) \ldots\left(1-\beta_{\mathrm{i}-1}\right) \int_{\mathrm{t}_{\mathrm{r}-1}}^{\mathrm{t}_{\mathrm{r}}} \mathrm{w}(\mathrm{x}) \mathrm{q}\left(\mathrm{t}_{\mathrm{i}}+\mathrm{z}-\mathrm{x}\right) \mathrm{dx}+\int_{\mathrm{t}_{\mathrm{i}-1}}^{\mathrm{t}_{\mathrm{i}}} \mathrm{w}(\mathrm{x}) \mathrm{q}\left(\mathrm{t}_{\mathrm{i}}+\mathrm{z}-\mathrm{x}\right) \mathrm{dx}\right\} \\
\text { and, } \quad & +\beta_{0} \int_{0}^{\mathrm{t}_{0}} \mathrm{w}(\mathrm{x}) \mathrm{q}\left(\mathrm{t}_{0}+\mathrm{z}-\mathrm{x}\right) \mathrm{dx}
\end{aligned}
$$

$$
\begin{aligned}
P\left(D=1 \mid T=t_{K}\right) & =\int_{t_{0}}^{t_{K} t} w(x) q(t-x) d x d t=\int_{0}^{t_{0}} w(x)\left[Q\left(t_{0}-x\right)-Q\left(t_{K}-x\right)\right] d x \\
& +\int_{t_{0}}^{t_{K}} w(x)\left[1-Q\left(t_{K}-x\right)\right] d x
\end{aligned}
$$

Here, $\mathrm{K}$ is the total number of screenings the individual will undergo in their lifetime, $t$ is the age of the individual at a screening exam, and $t_{0}$ is the age at which the individual has their initial screening. We define $\mathrm{w}(\mathrm{t}) \mathrm{dt}$ as the probability that an individual will transition from the disease-free state $\left(S_{0}\right)$ to the preclinical state $\left(S_{p}\right)$ during the interval $(\mathrm{t}, \mathrm{t}+\mathrm{dt}) ; \mathrm{q}(\mathrm{x})$ as probability distribution function of the sojourn time, $Q(z)=\int_{z}^{\infty} q(x) d x$ as the survivor function for the sojourn time, and $\beta=$ screening sensitivity. Screening sensitivity is defined as the number of patients that have cancer which is detected by screening divided by the total number of patients receiving screenings that have cancer. It is also knowns as the true positive rate $[16,17]$.

\section{Proposed methods}

We can apply the above methods to any screening schedule. For our example we consider three different initial screening ages for both men and women of $t_{0}=40,50$, and 60 . For each initial age we examine the effects of two different screening frequencies, $\Delta=1.0$ and 2.0 years. For each combination of initial screening age and screening frequency we consider two different mean sojourn times, MST $=2$ and 10 years. We also consider two different screening sensitivities for men $(0.3$ and $0.5)$ and two different sensitivities for women (0.8 and 0.9). We chose the value 0.3 for sensitivity for men due to the low sensitivity of prostate cancer screening and the value of 0.8 for sensitivity for women due to the high sensitivity of breast cancer. We then chose to include one more value for each gender that was close to the original value for effect, thus choosing 0.5 for men and 0.9 for women. The transition density function is that of a log normal $\left(\mu, \sigma^{2}\right)$ density function multiplied by 0.2 , the upper limit of lifetime risk of developing cancer,

$$
\mathrm{w}\left(\mathrm{t} \mid \mu, \sigma^{2}\right)=\frac{0.2}{\sqrt{2 \pi} \sigma \mathrm{t}} \exp \left\{-\frac{(\log t-\mu)^{2}}{2 \sigma^{2}}\right\}, \quad \sigma>0
$$

Most simulations done in cancer screening have used the Exponential distribution to describe the sojourn time distribution. We chose the log-logistic distribution as a means to explore a different option and because the log-logistic distribution includes an extra parameter that can account for over-dispersion as opposed to the exponential distribution. The log-logistic distribution yields the following survivor and hazard functions:

$$
\mathrm{Q}(\mathrm{x})=\frac{1}{1+(\mathrm{x} \rho)^{\mathrm{\kappa}}} \quad \text { and } \mathrm{h}(\mathrm{x})=\frac{\kappa \mathrm{x}^{\mathrm{\kappa}-1} \rho^{\kappa}}{1+(\mathrm{x} \rho)^{\mathrm{K}}}, \quad \kappa>0, \quad \rho>0
$$

We then combine these two functions to define the density function for the sojourn time: $\mathrm{q}(\mathrm{x})=\mathrm{h}(\mathrm{x}) \mathrm{Q}(\mathrm{x})$, where $\mathrm{x}$ is the sojourn time. The above equations contain the following unknown parameters, $\beta, \mu, \sigma^{2}, \kappa$, and $\rho$ (Table 2) [13].

The parameters, $\mu$ and $\sigma^{2}$, are used in the log normal distribution to describe the time duration in the preclinical state. We picked the

\begin{tabular}{|c|c|c|c|c|}
\hline \multirow{2}{*}{\multicolumn{2}{|c|}{ Parameter }} & \multirow[t]{2}{*}{ Definition } & \multicolumn{2}{|c|}{ Value(s) } \\
\hline & & & Men & Women \\
\hline \multicolumn{2}{|c|}{$\beta$} & Screening sensitivity & 0.3 and 0.5 & 0.8 and 0.9 \\
\hline \multicolumn{2}{|c|}{$\mu$} & $\begin{array}{l}\text { Location parameter for } \\
\text { transition density function } \\
\text { (log-normal) }\end{array}$ & 4.33 & 4.33 \\
\hline \multicolumn{2}{|c|}{$\sigma^{2}$} & $\begin{array}{l}\text { Scale parameter for } \\
\text { transition density function } \\
\text { (log-normal) }\end{array}$ & 0.17 & 0.17 \\
\hline \multicolumn{2}{|l|}{ K } & $\begin{array}{l}\text { Shape parameter for sojourn } \\
\text { time density function } \\
\text { (log-logistic) }\end{array}$ & 2.3 & 2.3 \\
\hline \multirow[t]{2}{*}{$\rho$} & $\begin{array}{l}\text { MST=2 } \\
\text { Years }\end{array}$ & \multirow{2}{*}{$\begin{array}{l}\text { Scale parameter for sojourn } \\
\text { time density function } \\
\text { (log-logistic) }\end{array}$} & 0.698 & 0.698 \\
\hline & $\begin{array}{l}\text { MST }=10 \\
\text { Years }\end{array}$ & & 0.1395 & 0.1395 \\
\hline
\end{tabular}
values for these parameters based on the fact that for most cancer, the transition from disease-free state to preclinical state has a mode around age 60 and a mean around age 70[13]. For the log logistic distribution, $\kappa$ was set at 2.3 because it must be greater than 2 for the second moment to exist, and from simulations run previously by $\mathrm{Wu}$ et al. it was usually around 2.3 [13]. Given $\kappa$ we were able to calculate $\rho$ setting the equation for the mean of a $\log$ logistic distribution equal to 2 and 10 .

\section{Results}

Results from all simulations are shown below. Figure 2 shows the conditional lifetime densities for the three different initial screening ages for both men and women. The following tables give the probability of no benefit (lead time equal to zero), for each initial screening age, screening frequency, and sojourn time distribution combination as

Table 2: Unknown parameters and values used in simulations. 
Citation: Kendrick SK, Rai SN, Wu D (2015) Simulation Study for the Sensitivity and Mean Sojourn Time Specific Lead Time in Cancer Screening When Human Lifetime is a Competing Risk. J Biom Biostat 6: 247. doi:10.4172/2155-6180.1000247

Page 4 of 7
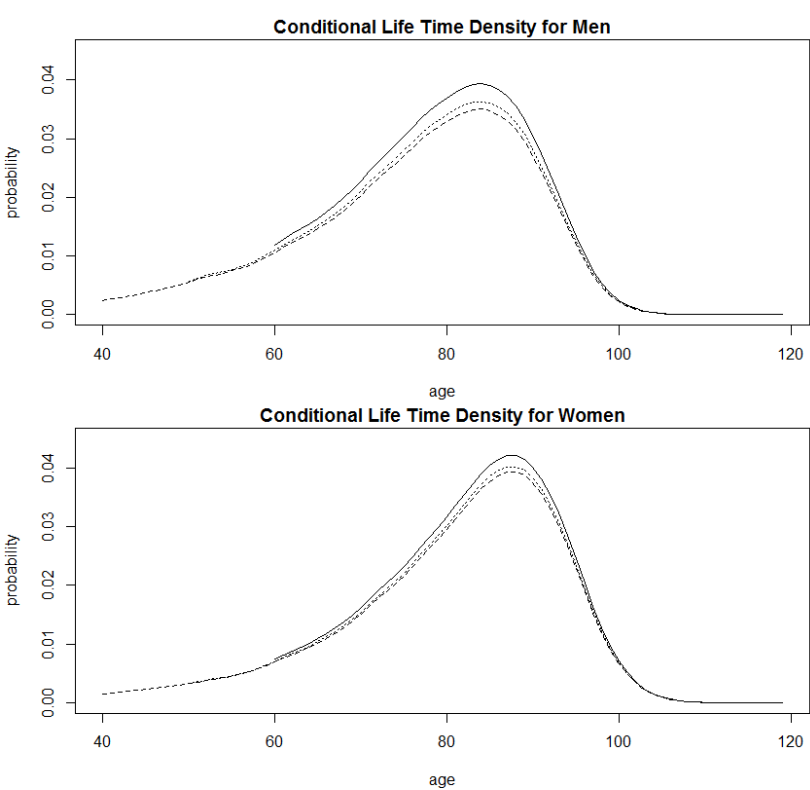

Figure 2: Conditional lifetime densities for initial screening ages of 40,50 , and 60 .

\begin{tabular}{|l|c|c|c|c|c|c|c|c|}
\hline \multicolumn{7}{|c|}{$\boldsymbol{\beta}=\mathbf{0 . 3}$} & \multicolumn{3}{|c|}{$\boldsymbol{\beta = 0 . 5}$} \\
\hline $\boldsymbol{\Delta}^{\mathbf{a}}$ & $\mathrm{p}_{0}^{b}$ & $\mathbf{1 - \mathbf { P } _ { \mathbf { 0 } }}$ & $\mathbf{E L}^{\mathbf{c}}$ & $\mathbf{M e d}^{\mathbf{d}}$ & $\mathrm{p}_{0}^{b}$ & $\mathbf{1 - P}_{\mathbf{0}}$ & $\mathbf{E L}^{\mathbf{c}}$ & Med $^{\mathbf{d}}$ \\
\hline Initial Screening Age $\mathbf{t}_{\mathbf{0}}=\mathbf{4 0}$ \\
\hline $\mathbf{1}$ Year & 51.81 & 48.19 & 4.57 & 3 & 31.44 & 68.56 & 3.96 & 3.2 \\
\hline 2 Years & 82.37 & 17.63 & 0.76 & 1.8 & 61.49 & 38.51 & 0.88 & 2.4 \\
\hline Initial Screening Age $\mathbf{t}_{\mathbf{0}}=\mathbf{5 0}$ & & & & & \\
\hline 1 Year & 51.49 & 48.51 & 4.92 & 2.1 & 31.51 & 68.49 & 4.49 & 2.1 \\
\hline 2 Years & 80.28 & 19.72 & 1.1 & 1.4 & 60.28 & 39.72 & 1.29 & 1.7 \\
\hline Initial Screening Age $\mathbf{t}_{\mathbf{0}}=\mathbf{6 0}$ & & & & & \\
\hline 1 Year & 51.33 & 48.67 & 4.11 & 1.9 & 31.84 & 68.16 & 3.99 & 1.9 \\
\hline 2 Years & 77.88 & 22.12 & 1.23 & 1.4 & 58.98 & 41.02 & 1.45 & 1.6 \\
\hline
\end{tabular}

aTime between screenings $\left(t_{i}-t_{i-1}\right)$

${ }^{\mathrm{b}} \mathrm{P}_{0}=\mathrm{P}(\mathrm{L}=0 \mid \mathrm{D}=1)=$ Probability of no early detection $(\%)$

${ }^{c}$ The mean lead time is in years

${ }^{d}$ The median when $L>0$ (for non-interval cases)

Table 3: A projection of the lead time distribution for men when mean sojourn time $=2$ years.

well as the estimate for the probability of benefit, the expected lead time, and the median lead time.

The above graphs show us that the conditional life time density for men and women are very similar. Therefore, even though we only examine screening sensitivities of 0.3 and 0.5 for men and 0.8 and 0.9 for women instead of examining all four screening sensitivities for both genders, we can still apply the results from men to women and vice versa without losing too much information (Tables 3-6).

Figures 3-6 give the density curves for the lead time for the different screening intervals, sojourn times, and initial screening ages for both men and women.

These results show that for cancer with a mean sojourn time (MST) of two years, an individual that begins screening at age 40 and receives screenings once a year, with screening sensitivity $=0.8$ has a

\begin{tabular}{|l|c|c|c|c|c|c|c|c|c|}
\hline \multicolumn{7}{|c|}{$\boldsymbol{\beta}=\mathbf{0 . 3}$} & \multicolumn{3}{c|}{$\boldsymbol{\beta = 0 . 5}$} \\
\hline $\mathbf{\Delta}^{\mathbf{a}}$ & $\mathrm{p}_{0}^{b}$ & $\mathbf{1 - \mathbf { P } _ { \mathbf { 0 } }}$ & $\mathbf{E L}^{\mathrm{c}}$ & $\mathbf{M e d}^{\mathbf{d}}$ & $\mathrm{p}_{0}^{b}$ & $\mathbf{1 - \mathbf { P } _ { \mathbf { 0 } }}$ & $\mathbf{E L}^{\mathbf{c}}$ & Med $^{\mathbf{d}}$ \\
\hline Initial Screening Age $\mathbf{t}_{\mathbf{0}}=\mathbf{4 0}$ \\
\hline $\mathbf{1}$ Year & 14.35 & 85.65 & 7.01 & 4.7 & 4.99 & 95.01 & 7.58 & 4.5 \\
\hline 2 Years & 28.32 & 71.68 & 3.72 & 4.1 & 12.68 & 87.32 & 4.45 & 4 \\
\hline Initial Screening Age $\mathbf{t}_{\mathbf{0}}=\mathbf{5 0}$ & & & & & \\
\hline 1 Year & 16.85 & 83.15 & 7.61 & 4 & 6.63 & 93.37 & 8.5 & 3.8 \\
\hline 2 Years & 30.49 & 69.51 & 4.55 & 3.4 & 14.63 & 85.37 & 5.54 & 3.3 \\
\hline Initial Screening Age $\mathbf{t}_{\mathbf{0}}=\mathbf{6 0}$ & & & & & \\
\hline 1 Year & 20.69 & 79.31 & 6.93 & 3.6 & 9.12 & 90.88 & 7.92 & 3.4 \\
\hline 2 Years & 34.12 & 65.88 & 4.56 & 3 & 17.67 & 82.33 & 5.64 & 3 \\
\hline
\end{tabular}

aTime between screenings $\left(t_{-}-t_{-}-1\right)$

${ }^{b} P_{0}=P(L=0 \mid D=1)=$ Probability of no early detection (\%)

'The mean lead time is in years

dThe median when $\mathrm{L}>0$ (for non-interval cases)

Table 4: A projection of the lead time distribution for men when mean sojourn time $=10$ years.

\begin{tabular}{|c|c|c|c|c|c|c|c|c|}
\hline & \multicolumn{4}{|c|}{$\beta=0.8$} & \multicolumn{4}{|c|}{$\beta=0.9$} \\
\hline & \multicolumn{8}{|c|}{$K=2.3 \rho=0.698$} \\
\hline$\Delta^{\mathrm{a}}$ & $\mathrm{p}_{0}^{b}$ & $1-P_{0}$ & $E^{c}{ }^{c}$ & Med $^{d}$ & $\mathrm{p}_{0}^{b}$ & $1-P_{0}$ & $\mathrm{EL}^{\mathrm{c}}$ & Med $^{d}$ \\
\hline \multicolumn{9}{|c|}{ Initial Screening Age $t_{0}=40$} \\
\hline 1 Year & 15.3 & 84.7 & 2.09 & 4.5 & 12.65 & 87.35 & 1.69 & 4.4 \\
\hline 2 Years & 40.08 & 59.92 & 0.73 & 4.1 & 35.27 & 64.73 & 0.75 & 4.2 \\
\hline \multicolumn{9}{|c|}{ Initial Screening Age $t_{0}=50$} \\
\hline 1 Year & 15.3 & 84.7 & 3.08 & 2.1 & 12.55 & 87.45 & 1.9 & 2 \\
\hline 2 Years & 39.5 & 60.5 & 1.29 & 2.1 & 34.69 & 65.31 & 1.7 & 2 \\
\hline \multicolumn{9}{|c|}{ Initial Screening Age $t_{0}=60$} \\
\hline 1 Year & 15.42 & 84.58 & 3.29 & 1.7 & 12.51 & 87.49 & 2.97 & 1.6 \\
\hline 2 Years & 38.87 & 61.13 & 1.6 & 1.7 & 34.03 & 65.97 & 1.66 & 1.6 \\
\hline
\end{tabular}

aTime between screenings $\left(t_{-}-t_{-}-1\right)$

${ }^{\mathrm{b}} \mathrm{P} 0=\mathrm{P}(\mathrm{L}=0 \mid \mathrm{D}=1)=$ Probability of no early detection $(\%)$

'The mean lead time is in years

'The median when $L>0$ (for non-interval cases)

Table 5: A projection of the lead time distribution for women when mean sojourn time $=2$ years.

\begin{tabular}{|l|c|c|c|c|c|c|c|c|}
\hline \multicolumn{7}{|c|}{$\boldsymbol{\beta}=\mathbf{0 . 8}$} & \multicolumn{3}{c|}{$\boldsymbol{\beta}=\mathbf{0 . 9}$} \\
\hline $\mathbf{\Delta}^{\mathbf{a}}$ & $\mathrm{p}_{0}^{b}$ & $\mathbf{1 - \mathbf { P } _ { \mathbf { 0 } }}$ & $\mathbf{E L}^{\mathbf{c}}$ & $\mathbf{M e d}^{\mathbf{d}}$ & $\mathrm{p}_{0}^{b}$ & $\mathbf{1 - P}_{\mathbf{0}}$ & $\mathbf{E L}^{\mathbf{c}}$ & Med $^{\mathbf{d}}$ \\
\hline Initial Screening Age $\mathbf{t}_{\mathbf{0}}=\mathbf{4 0}$ \\
\hline 1 Year & 1.02 & 98.98 & 6.9 & 4.6 & 0.64 & 99.36 & 7.09 & 4.4 \\
\hline 2 Years & 3.59 & 96.41 & 4.25 & 4.3 & 2.53 & 97.47 & 1.39 & 4.2 \\
\hline Initial Screening Age $\mathbf{t}_{\mathbf{0}}=\mathbf{5 0}$ \\
\hline 1 Year & 1.42 & 98.58 & 9.27 & 3.6 & 0.8 & 99.2 & 9.65 & 3.5 \\
\hline 2 Years & 4.14 & 95.86 & 6.31 & 3.3 & 2.72 & 97.28 & 2.32 & 3.2 \\
\hline Initial Screening Age $\mathbf{t}_{\mathbf{0}}=\mathbf{6 0}$ & & & & & \\
\hline 1 Year & 2.06 & 97.94 & 9.29 & 3.2 & 1.09 & 98.91 & 9.7 & 3.1 \\
\hline 2 Years & 5.07 & 94.93 & 6.8 & 2.9 & 3.09 & 96.91 & 2.8 & 2.8 \\
\hline
\end{tabular}

aTime between screenings $\left(t_{i}-t_{i}-1\right)$

${ }^{\mathrm{b}} \mathrm{P}_{0}=\mathrm{P}(\mathrm{L}=0 \mid \mathrm{D}=1)=$ Probability of no early detection (\%)

cThe mean lead time is in years

dThe median when $L>0$ (for non-interval cases)

Table 6: A projection of the lead time distribution for women when mean sojourn time $=10$ years. 
Citation: Kendrick SK, Rai SN, Wu D (2015) Simulation Study for the Sensitivity and Mean Sojourn Time Specific Lead Time in Cancer Screening When Human Lifetime is a Competing Risk. J Biom Biostat 6: 247. doi:10.4172/2155-6180.1000247

Page 5 of 7
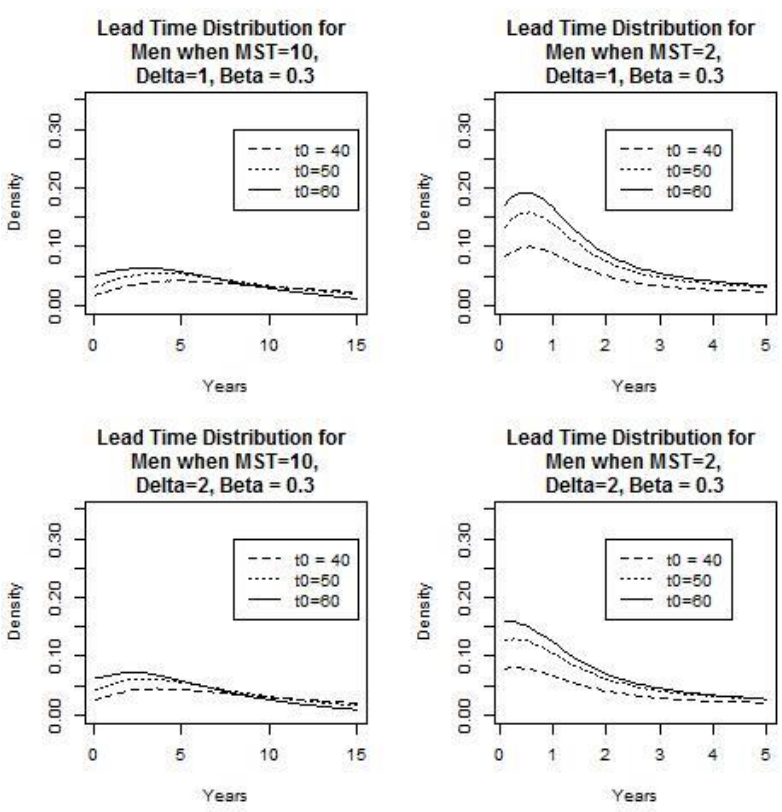

Figure 3: Lead time probability distribution for men with initial screening ages of 40,50 , and 60 , mean sojourn times of 2 years and 10 years, and $\beta=0.3$.
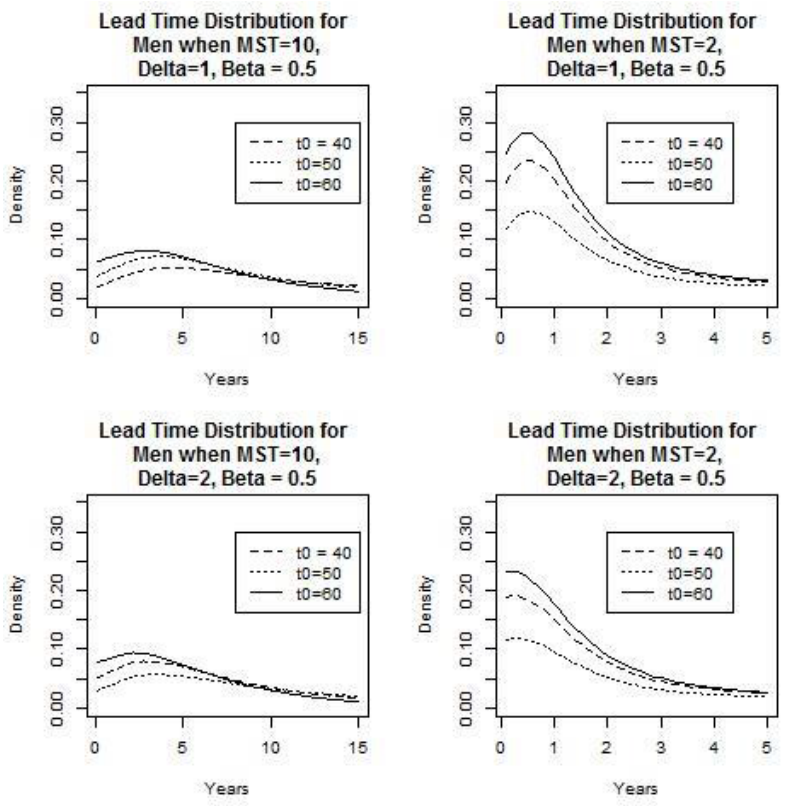

Figure 4: Lead time probability distributions for men with initial screening ages of 40,50 , and 60 , mean sojourn times of 2 years and 10 years, and $\beta=0.5$.

84.7\% chance that their cancer will be detected by screening. This value decreases to $59.9 \%$ when screening frequency is decreased to once every two years. We see a similar trend for initial screening ages of 50 years, $84.7 \%$ and $60.5 \%$, and 60 years, $84.5 \%$ and $61.1 \%$, for $\Delta=1.0$ and 2.0 years respectively. We find that the probability an individual will experience detection by screening decreases as screening frequency decreases when we hold MST and age constant. This probability remains relatively constant as age increases when holding screening frequency and MST constant, increases as MST increases with age and screening frequency held constant, and increases as sensitivity increases.

From the graphs of the lead time distributions based on the loglogistic sojourn time distribution, we see that the mode, mean, and median lead time are monotonically increasing as sensitivity increases and as MST increases. We also find that the mean and mode lead time decrease as screening frequency decreases with MST and age held
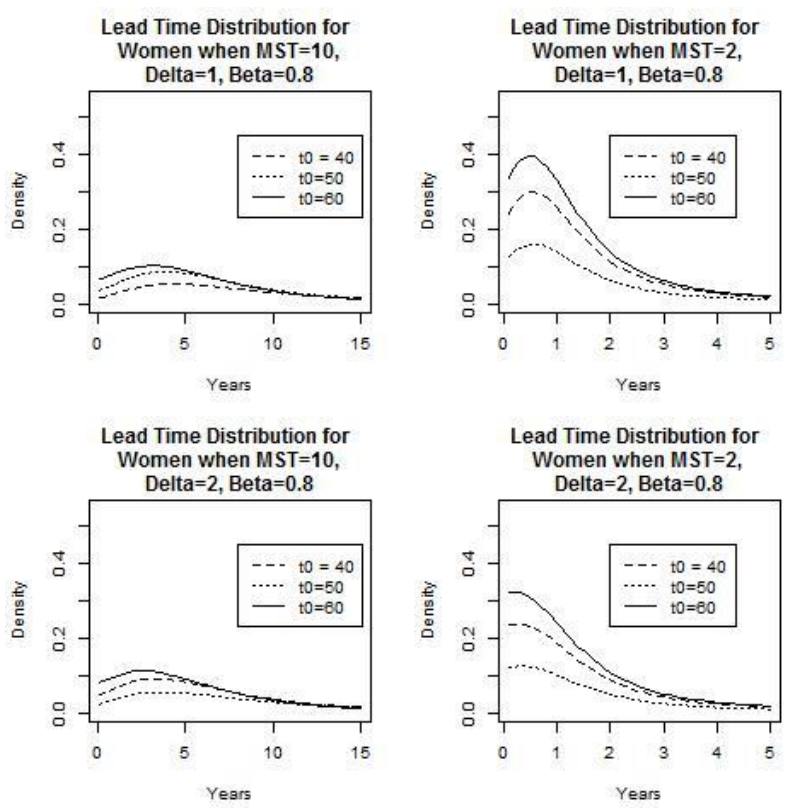

Figure 5: Lead time probability distributions for women with initial screening ages of 40,50 , and 60 , mean sojourn times of 2 years and 10 years, and $\beta=0.8$.
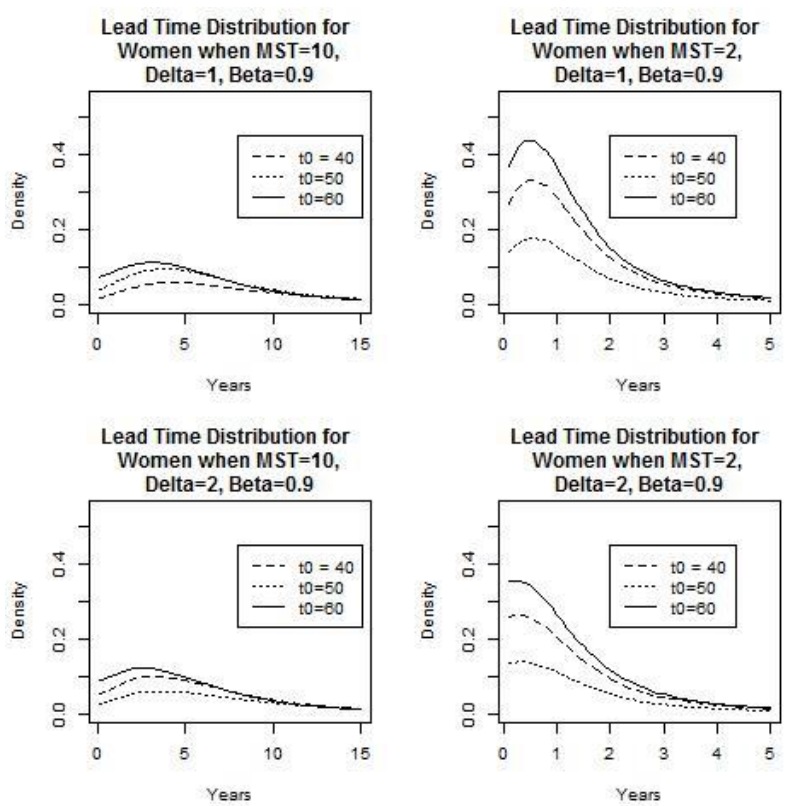

Figure 6: Lead time probability distributions for women with initial screening ages of 40,50 , and 60 , mean sojourn times of 2 years and 10 years, and $\beta=0.9$. 
constant. Also, it is important to note that mean and mode lead time increase as age increases with MST and screening frequency being held constant. The trends we see in the median lead time are that it increases as age increases (frequency and MST held constant) and decreases as screening frequency decreases (MST and age held constant).

\section{Discussion and Conclusion}

We carried out extensive simulation studies using the lead time model derived in Wu et al. (2012). The purpose was to explore how lead time changes when other factors, such as sensitivity, sojourn times, transition probability, initial age, and screening frequency change.

We examined the lead time distribution for a log-logistic sojourn time distribution, two different mean sojourn times: 2 years and 10 years, and two different screening frequencies: once a year and every two years, for both men and women. For men we looked at screening sensitivities $=0.3$ and 0.5 whereas we used sensitivities of 0.8 and 0.9 for women. We chose these sensitivities because cancers that are found only in men (i.e. prostate cancer) have relatively low screening sensitivities whereas those found only in women (i.e. breast cancer) have relatively high screening sensitivities. We also found that the outcomes were very similar for both men and women, and therefore the results based on the screening sensitivities for men can be applied to women and vice versa.

Our simulations showed that as the sensitivity of screening increases, the probability of detecting disease by screening increases. For instance, our results revealed that for cancer with a mean sojourn time of 2 years, an individual that begins annual screenings at age 40 has a $48.2 \%$ chance of being detected by screening if the sensitivity is 0.3 . This probability increases to $68.6 \%$ when the sensitivity is increased to 0.5 . We see similar results for initial screening ages of 50 years, $48.5 \%$ and $68.5 \%$, and 60 years, $48.7 \%$ and $68.2 \%$, for sensitivity $=0.3$ and 0.5 , respectively. When mean sojourn time is increased to 10 years, a greater sensitivity still yields a greater probability of early detection, however the difference is not as drastic. As an example, an individual beginning annual screenings at age 40 has an $85.7 \%$ probability of early detection by screening when sensitivity is 0.3 and a $95.0 \%$ probability when sensitivity is increased to 0.5 . The trend of increased sensitivity leading to increased chance of early detection is seen across all screening intervals, all initial screening ages, all sensitivity levels, and all mean sojourn times, for both men and women.

Gathering data from all of these simulations allows us to extend our results to several prevalent cancers such as breast cancer, prostate cancer, lung cancer, and colorectal cancer which are some of the most common cancers amongst men and/or women today. Wu et al. examined the age dependent screening sensitivity in breast cancer. Their results showed mammography to have a posterior mean sensitivity of 0.74 with a $90 \%$ credible interval of $(0.43,0.97)$ [13]. Lung cancer and colorectal cancer were found to have similar mean sojourn times as breast cancer when assuming an exponentially distributed sojourn time. Lung cancer showed mean sojourn times between 1.38 and 3.86 years and sensitivity with $95 \%$ confidence interval $0.89(0.72$, $0.98)[8,18]$; proximal colorectal cancer gave mean sojourn times of 3.86 years for individuals aged 45 to 54 years, 3.78 for 55 to 64 year olds, and 2.70 for 65-74 year olds; and distal colorectal cancer was found to have mean sojourn times of 3.35 for individuals aged 45 to 54 years, 2.24 for 55 to 64 year olds, and 2.10 for 65 to 74 year olds; and an overall sensitivity estimate of about $0.75(0.45,0.97)$ for colonoscopies and $0.75(0.65,0.84)$ for fecal occult blood tests $[9,10,19]$. A doctor could use our results where MST is two years to develop an efficient screening program for individuals aged 40 to 60 years old who are at risk for breast cancer (use sensitivity $=0.8$ or 0.9 ), lung cancer (use sensitivity $=0.8$ or 0.9 ), or colorectal cancer (use sensitivity $=0.8$ ). Another study found prostate cancer has a mean sojourn time between 11.3 and 12.6 years with a sensitivity around $21 \%$ for prostate specific antigen tests and $53 \%-59 \%$ for digital rectal exams [14]. In this case, our results where MST is equal to 10 and sensitivity is either 0.3 or 0.5 could be used to come up with effective screening programs for men at risk for prostate cancer.

Our simulations only take age and gender into account as a covariate. In the future, we hope to not only get more accurate estimates for the aforementioned parameters and distributions, but also to include some other possible covariates to make our results applicable to more subgroups. It is also important to note that in reality individuals do not go for their screening at exact one or two year intervals but instead somewhere within an interval around the one or two year mark. Even though our model can handle irregular screening intervals, we did not explore this but it can be explored in future work. Another option for future work is to use a model where the sensitivity, $\beta$, is a function of age instead of a fixed value. Finally, our model assumes that sensitivity and sojourn time are independent of each other even though there is evidence that suggests this may not be the case. In future work, we may want to examine this relationship more closely.

\section{Acknowledgement}

Dr. Rai was partly supported by Wendell Cherry Chair in Clinical Trial Research and by Dr. Miller, Director of James Brown Cancer Center, University of Louisville.

\section{References}

1. Centers for Disease Control and Prevention (2009) United States Cancer Statistics (USCS), USA.

2. Zelen M, Feinleib M (1969) On the theory of screening for chronic diseases Biometrika 56: 601-614.

3. Prorok PC (1982) Bounded Recurrence Times and Lead Time in the Design of a Repetitive Screening Program. Journal of Applied Probability 19: 10-19.

4. U.S. Preventative Services Task Force (2012) Screening for Prostate Cancer

5. Centers for Disease Control and Prevention (2012) Breast Cancer.

6. Centers for Disease Control and Prevention (2011) Lung Cancer Screening

7. Howlader NNA, Krapcho M, Garshell J, Miller D, Altekruse SF, et al. (2014) SEER Cancer Statistics Review, 1975-2011, National Cancer Institute.

8. Wu D, Erwin D, Rosner GL (2011) Sojourn time and lead time projection in lung cancer screening. Lung Cancer 72: 322-326.

9. Church TR, Ederer F, Mandel JS (1997) Fecal occult blood screening in the Minnesota study: sensitivity of the screening test. J Natl Cancer Inst 89: 14401448.

10. Halligan S, Altman DG, Taylor SA, Mallett S, Deeks JJ, et al. (2005) CT colonography in the detection of colorectal polyps and cancer: systematic review, meta-analysis, and proposed minimum data set for study leve reporting. Radiology 237: 893-904.

11. Wu D, Erwin D, Rosner GL (2009) Estimating key parameters in FOBT screening for colorectal cancer. Cancer Causes Control 20: 41-46.

12. Kavanagh AM, Giles GG, Mitchell H, Cawson JN (2000) The sensitivity, specificity, and positive predictive value of screening mammography and symptomatic status. J Med Screen 7: 105-110.

13. Wu D, Rosner GL, Broemeling L (2005) MLE and Bayesian inference of agedependent sensitivity and transition probability in periodic screening. Biometrics 61: 1056-1063.

14. Wilbur J (2008) Prostate cancer screening: the continuing controversy. Am Fam Physician 78: 1377-1384.

15. Thompson IM, Ankerst DP, Chi C Lucia MS, Goodman PJ, et al. (2005) Operating characteristics of prostate-specific antigen in men with an initial PSA 
Citation: Kendrick SK, Rai SN, Wu D (2015) Simulation Study for the Sensitivity and Mean Sojourn Time Specific Lead Time in Cancer Screening When Human Lifetime is a Competing Risk. J Biom Biostat 6: 247. doi:10.4172/2155-6180.1000247

level of $3.0 \mathrm{ng} / \mathrm{ml}$ or lower. JAMA 294: 66-70

16. Wu D, Kafadar K, Rosner GL, Broemeling LD (2012) The lead time distribution when lifetime is subject to competing risks in cancer screening. Int J Biostat 8.

17. Wu D, Rosner GL, Broemeling LD (2007) Bayesian inference for the lead time in periodic cancer screening. Biometrics 63: 873-880.
18. Chien CR, Chen TH (2008) Mean sojourn time and effectiveness of mortality reduction for lung cancer screening with computed tomography. Int $\mathrm{J}$ Cancer 122: 2594-2599.

19. Zheng W, Rutter CM (2012) Estimated mean sojourn time associated with hemoccult SENSA for detection of proximal and distal colorectal cancer. Cancer Epidemiology, Biomarkers and Prevention 21: 1722-1730. 